\title{
Long-Term Trends in the Survival of Women with Endometrial Cancer in Canada: A Population-Based Study ${ }^{*}$
}

\author{
Laurie Elit ${ }^{1,2 \#}$, Alice Lytwyn ${ }^{1,3}$, Noori Akhtar-Danesh ${ }^{1,4}$ \\ ${ }^{1}$ Department of Clinical Epidemiology \& Biostatistics, McMaster University, Hamilton, Canada; ${ }^{2}$ Department of Obstetrics and Gy- \\ necology, McMaster University, Hamilton, Canada; ${ }^{3}$ Department of Pathology and Molecular Medicine, McMaster University, Ham- \\ ilton, Canada; ${ }^{4}$ School of Nursing, McMaster University, Hamilton, Canada. \\ Email: " laurie.elit@jcc.hhsc.ca
}

Received April 20 $0^{\text {th }}, 2012$; revised May 23 ${ }^{\text {rd }}, 2012$; accepted June $6^{\text {th }}, 2012$

\begin{abstract}
Introduction: Annually in Canada, endometrial cancer affects approximately 4500 women and 790 are expected to die of their disease. To better understand survival trends across the country we undertook this population based study of Canadian women diagnosed with endometrial cancer. Long term trends in relative survival were evaluated by age and geographic region of residence. Methods: Women with an ICD-10 code of C54 and endometrial cancer were identified from the Canadian Cancer Registry. They were included if the incident diagnosis occurred between 1992 and 2005, and they were 16 years and older at diagnosis. A flexible parametric model was used to determine relative survival ratio (i.e., the observed survival rate among cancer patients divided by the expected survival rate in the general population). Results: 18,486 women were diagnosed with endometrial cancer. Mean age was $63.4(\mathrm{SD}=11.8)$ year. Relative survival decreased with each successive age group cohort of patient $(16-49$ yr compared to over 60 years, $p<0.001)$. When relative survival was adjusted for age, women in British Columbia had the best outcomes (eastern Canada compared to other jurisdictions $p<0.001$ ). Five-year survival outcomes improved for each age group cohort during the 1992 to 2005 time frame. Conclusions: Regional variations in relative survival were identified across Canada for women with endometrial cancer. This suggests that other factors related to the patient or processes of care are involved. Examining these factors in further detail may provide opportunities to improve the care of women with endometrial cancer in Canada.
\end{abstract}

Keywords: Endometrial Cancer; Relative Survival

\section{Introduction}

Uterine cancer is the most common gynaecological cancer. The Canadian Cancer Society reports that uterine cancer affects about 4500 women across Canada annually, and about 790 women are expected to die from this disease every year [1]. Trends in incidence and survival over time and across jurisdictions can suggest where patterns of care may offer benefit. Population studies provide an opportunity to measure outcomes variables such as overall survival and disease free survival, while adjusting for variations in patient factors (i.e., age, weight) [2]. Such information can be highly relevant to clinicians, patients and health administrators. For instance, regional variations may inform strategies for more optimal care delivery patterns. Investigation of potential health care delivery factors may help differentiate between those that

\footnotetext{
*Conflict of interest statement: the authors declare that there are no conflicts of interest.

${ }^{\#}$ Corresponding author.
}

do or do not appear to impact on out-come. For example, in 2007, Kwon et al. [3] showed that wait times for endometrial cancer surgery in Ontario increased, but their data did not show any impact on outcome.

Uterine cancer is made up of malignancies arising from the epithelial cells (otherwise known as endometrial cancer) or the stromal cells (i.e., leimyosarcoma, endometrial stromal sarcoma, mixed mesodermal tumor to name a few) or metastatic to the uterus (i.e., lymphoma). Endometrial cancer is the most common subtype of uterine cancer. In this paper we undertake to describe the survival patterns for women diagnosed with endometrial cancer in Canada over the period of 1992-2005. We examine whether long-term trends in relative survival are dependent on patient age and geographic region of residence.

\section{Patients and Methods}

We identified women with endometrial cancers in the 
Canadian Cancer Registry (CCR) dataset using the International Statistical Classification of Diseases and Related Health Problems, Tenth Revision (ICD-10). First we indentified tumours with codes C54.0 (Isthmus uteriincluding lower uterine segment), C54.1 (Endometrium), C54.2 (Myometrium), C54.3 (Fundus uteri), C54.8 (Overlapping lesion of corpus uteri), and C54.9 (Corpus uteri, unspecified). Then, using the International Classification of Diseases for Oncology (ICD-O-2 or ICD-O-3) codes, the analysis was limited to patients with only endometrial adenocarcinoma [4]. Patients were excluded if the diagnosis was only based on the death certificate or autopsy. Women were included if they had a new diagnosis of endometrial cancer from 1992-2005 and were between 16 years or older at the time of diagnosis. Follow-up information was retained until the end of 2006.

For the analysis by age, women were grouped into strata given their age at diagnosis $(16$ - 49, 50 - 59, 60 $69,70-79$, and 80 and over).

The geographical region was identified based on the patient's postal code at time of diagnosis. Given that health care in Canada is provincially funded, women were grouped based on the province at the time of diagnosis. In addition, because of small sample size some provinces were collapsed into geographically cohesive regions. The resulting geographic regions from west to east were: 1) British Columbia; 2) central-west and northern Canada: Alberta, Saskatchewan, Manitoba, Yukon, Nunavut, Northwest Territories; 3) Ontario; and 4) eastern Canada: New Brunswick, Nova Scotia, Prince Edward Island and Newfoundland. Data for the province of Quebec were excluded because the data on deaths was not available.

\section{Statistical Analysis}

It has become standard to use relative survival analysis for population-based cancer registry datasets $[5,6]$. Relative survival ratio (or simply relative survival) is defined as the observed survival rate among cancer patients divided by the expected survival rate in the general population of the same age and sex. It shows the extent to which cancer shortens life $[7,8]$. The advantage of relative survival is that there is no need to know the actual cause of death, although it includes all causes directly or indirectly associated with the diagnosis of cancer [7]. It is often age-adjusted to account for the fact that the risk of death increases as we age and the population is aging over time.

We used the flexible parametric model $[9,10]$ to estimate the relative survival ratio for different age groups and regions. To estimate the relative survival, the background mortality rate for the general population (the rate at which the death occurs in the general population) was incorporated in the model. The background mortality rate is usually derived from the country's life-table. We retrieved an abridged 5-year background mortality rate from the Statistics Canada website [11].

First, we fitted two separate models; one for each of the independent variables of age group and the geographical region. Then, we incorporated age group, geographical region, and year of diagnosis into a final statistical model using a stepwise forward approach to estimate the relative survival where the effect of each variable was adjusted for the effects of the other variables. To include year of diagnosis in the model, a restricted cubic splines with five knots was used. Restricted cubic splines [12] adds more flexibility to the potential nonlinear relationship between relative survival and year of diagnosis. The likelihood ratio test was used to compare between different models. After fitting the final model we predicted the two- and five-year relative survival ratio for each age group and region based on the year of diagnosis [13].

The flexible parametric model was fitted using the freely available stpm 2 software developed by Lambert and Royston [9] for the Stata package. Descriptive statistical analysis was conducted using Stata/SE 12.0 (Stata Corporation, College Station, TX).

\section{Results}

A total of 18,486 women were identified with endometrial cancer between 1992 and 2005. Their mean age at diagnosis was $63.4(\mathrm{SD}=11.8)$ year (median 63 years). Table 1 shows the age distribution at diagnosis and the number of deaths among endometrial cancer patients for each age group by geographic region. Only $11.1 \%$ of women were pre-menopausal (using age less than 50 as a proxy for premenopausal status) at time of diagnosis and $88.9 \%$ were 50 years old and over. Over half of all the endometrial cancers were diagnosed in Ontario. This is almost equal to the proportion of females in the general population in Ontario compared to the rest of Canada (excluding Quebec) [13]. The highest rate of death (21.6\%) was noted in Eastern Canada compared to Ontario (20.3\%), British Columbia (14.0\%; the lowest rate), and Central-West \& Northern Canada (18.9\%). In total 3591 patients (19.4\%) diagnosed with endometrial cancer died by the end of 2006 .

Relative survival by age group is shown in Figure 1. Relative survival clearly decreased with age. Women under 50 years had a statistically superior relative survival compared to women 60 years and old $(\mathrm{p}<0.001)$.

The relative survival ratios for the geographic regions are presented in Figure 2. While the curves are relatively close, relative survival is lower for Eastern Canada compared to the other regions $(p<0.001)$. As can be seen, British Columbia has the highest relative survival compared to the other geographical regions. These differ- 
Table 1. Distribution of age and death based on the province at the time of diagnosis for endometrial cancer patients.

\begin{tabular}{|c|c|c|c|c|c|c|c|c|c|c|}
\hline & \multicolumn{2}{|c|}{ Ontario } & \multicolumn{2}{|c|}{ British Columbia } & \multicolumn{2}{|c|}{ Eastern Canada } & \multicolumn{2}{|c|}{$\begin{array}{c}\text { Central-West \& Northern } \\
\text { Canada }\end{array}$} & \multicolumn{2}{|c|}{ Total } \\
\hline & n (\%) & \# of death & n (\%) & $\#$ of death & n $(\%)$ & $\#$ of death & n (\%) & \# of death & n $(\%)$ & \# of death \\
\hline \multicolumn{11}{|l|}{ AGE GROUP } \\
\hline$<50$ year & $1100(10.4)$ & 92 & $228(11.4)$ & 10 & $204(12.5)$ & 23 & $515(12.2)$ & 34 & 2047 (11.1) & 159 \\
\hline 50 - 59 year & $2943(27.7)$ & 282 & $567(28.3)$ & 29 & $471(28.9)$ & 42 & $1220(28.8)$ & 103 & $5201(28.1)$ & 456 \\
\hline 60 - 69 year & $3168(29.8)$ & 557 & $617(30.8)$ & 76 & $436(26.8)$ & 84 & 1215 28.7) & 204 & $5436(29.4)$ & 921 \\
\hline 70 - 79 year & $2450(23.1)$ & 753 & $391(19.5)$ & 85 & $361(22.2)$ & 116 & $921(21.8)$ & 256 & $4123(22.3)$ & 1210 \\
\hline$\geq 80$ year & $957(9.0)$ & 474 & $201(10.0)$ & 80 & $157(9.6)$ & 87 & $364(8.6)$ & 204 & $1679(9.1)$ & 845 \\
\hline Total & 10,618 & 2158 & 2004 & 280 & 1629 & 352 & 4235 & 801 & 18,486 & 3591 \\
\hline
\end{tabular}

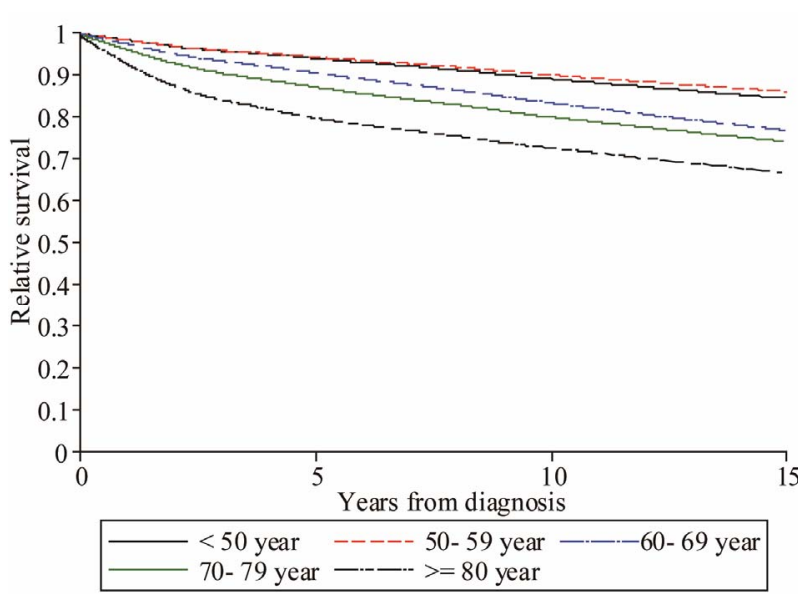

Figure 1. Relative survival based on age group at diagnosis.

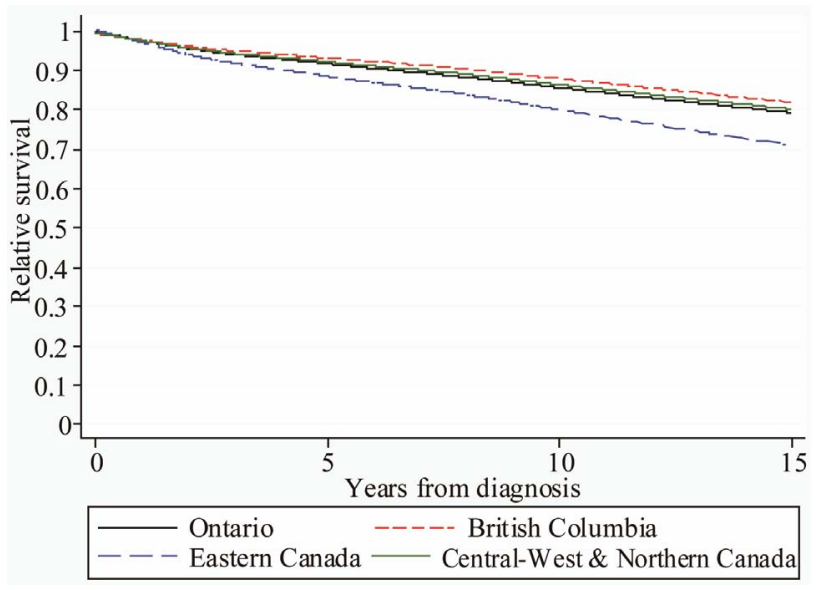

Figure 2. Relative survival based on the geographical region.

ences remain statistically significant even when adjusted by age group.

The results from fitting a flexible parametric model including age group, the geographical region, and year of

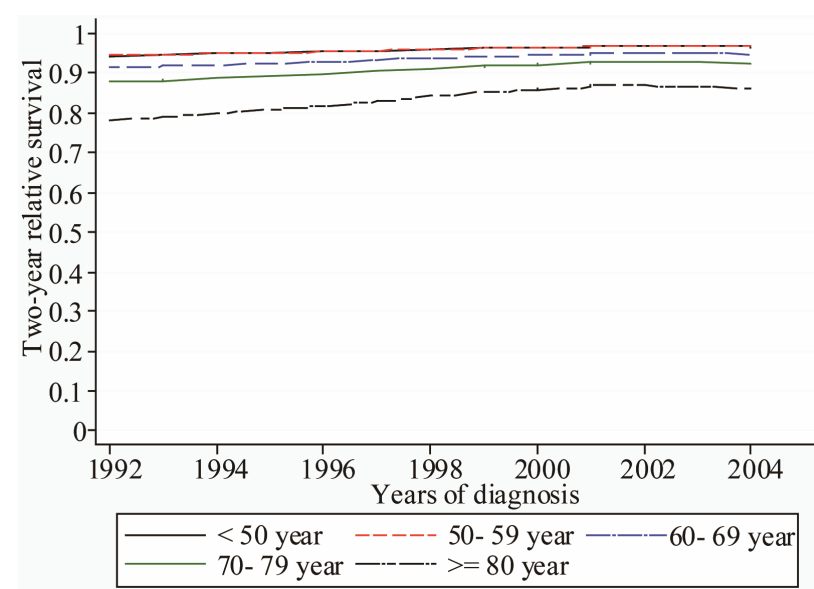

Figure 3. Trends in two-year relative survival based on age group in patients diagnosed with endometrial cancer in Canada during 1992-2005 with follow-up to 2006.



Figure 4. Trends in five-year relative survival based on age group in patients diagnosed with endometrial cancer in Canada during 1992-2005 with follow-up to 2006.

diagnosis are also presented as two- and five-year relative survival ratio based on year of diagnosis for different 
age groups (Figures 3 and $\mathbf{4})$. These figures indicate that, in general, two- and five-year survival for endometrial cancer patients improved for all age groups over the period of 1992-2005.

\section{Discussion}

To our knowledge, this is the first study using the flexible parametric model to estimate long-term relative survival for endometrial cancer in a population-based study. Use of flexible parametric models in survival analysis is quite new; although the theoretical paper was published by Royston and Parmar in 2002 [10]; the actual "how to do" paper was published in 2009 by Lambert and Royston [9]. We chose a flexible parametric model because it is more flexible and more powerful compared to the other methods in mimicking the actual trends in mortality (hazard rate) and survival pattern in datasets.

In year 2000 the overall 5-year relative survival in the Canadian population was well above $80 \%$ for all age groups for the patients diagnosed with endometrial cancers (Figure 4) and the average 5-year relative survival was $89.4 \%$. This is in keeping with the age standardized values quoted in the literature [14-17]. This is higher than the Cancer System Quality Index (CSQI) Cancer Care Ontario reported rate of $83 \%$ for 1994-1998 and 2004-2008. This later rate was for all histologic types of cancer involving the uterus.

As Lee et al. [18] pointed out, endometrial cancer is primarily known as a postmenopausal disease and it is uncommon to find endometrial cancer in women during their reproductive years. In Lee's work, less than $15 \%$ of endometrial cancer patients were premenopausal [18]. In our study $11.1 \%$ of women with endometrial cancer were premenopausal which included over 2000 women less than 50 years of age. Other studies that focused on endometrial cancer in the reproductive aged women have small numbers of women. Our analysis corroborates a survival benefit for the reproductive age group compared to older aged women.

We found that relative survival is highly dependent on the age of diagnosis. We showed that relative survival decreases with increasing age and this has also been seen in the USA $[19,20]$ and the Nordic Countries [16]. This may in part be related to higher rate of co-morbidities in older women, earlier diagnosis of endometrial cancer in younger women because of indicators such as changes in menstrual function or increased self awareness (i.e., body image), as well as more aggressive histological types in older women.

Over the period of 1992-2006 there has been a general drift toward improving survival time over all age groups. Our analysis indicates that two- and five-year survivals have risen slightly over this time frame. Similar trends have been reported in the USA [19], Japan [21], UK [22] and the Nordic countries [16]. In part this may reflect shorter wait times and better diagnostic techniques to identify endometrial cancer and thus down staging, improved anaesthesiology and postoperative care, improved therapies for uterine cancer, access to several lines of adjuvant chemotherapy and biologic agents, and access to palliative care (i.e., less postoperative mortality after a palliative bowel resection).

This is the first report that compares relative survival ratio for endometrial cancer among geographic regions of Canada which identified a higher relative survival ratio in British Columbia compared to the other regions (Figure 2). This difference remained the same after adjusting for age group. This may reflect variations in patient factors (i.e., body mass index, ethnicity) or process issues (i.e., access to diagnostic tests, access to surgery, opportunity for subsequent lines of chemotherapy or biologic agents).

The strengths of this work include the high quality data as it is nationwide and population-based. In addition, we analyzed information from a large population (more than 18,000 women over fifteen years (1992-2006)).

A notable limitation of this paper is that we were unable to adjust the analysis based on the stage or grade of tumours as this information is not currently available from CCR dataset. Access to other patient information like race $[19,20]$ size of dwelling and marital status [15], status of disease details like stage and grade $[20,23,24]$, histology [24], treatment information like details of the surgical intervention $[23,24]$, subsequent treatment [2325], could help focus in on the potential reason(s) for variations in outcome that are identified. Another limitation is that we used the general survival for the Canadian population as our standard for survival. Survival may vary by geographic region and thus general survival for region may have been a superior method. Unfortunately, this information is not available. Another limitation is that the model combines cancer related death rather than cause specific death i.e., death from cancer versus death from treatment complication. This finer level of information was not available to us. Another limitation is that administrative databases were never designed for population based studies. Thus any results are hypothesis generating and require further investigation by other methods to validate the hypothesis.

\section{Conclusion}

This work indicates that in Canada, when relative survival is used to measure outcome, both patient age at diagnosis and geographic region of residence affect outcome. When adjusted for age at diagnosis, geographic region of patient residence affects continues to influence. Evaluating other factors either related to disease (i.e., histological type), patient (i.e., body mass index) or proc- 
esses of care (i.e., wait times for surgery) may act as confounders in the variations in relative survival seen by region. If differences in relative survival by region persist despite controlling for such confounders, this may suggest opportunities to improve care.

\section{Acknowledgements}

The authors wish to thank the Canadian Cancer Registry and Statistics Canada for allowing us access to the dataset. We also wish to thank the staff of the Research Data Centre, McMaster University for their help.

\section{REFERENCES}

[1] Canadian Cancer Society, "Canadian Cancer Statistics 2010," Author, Toronto, 2010.

[2] D. S. McMeekin, K. M. Alektiar, P. J.Sabbatini and R. J. Zaino, "Corpus: Epithelial Tumors," In: R. R. Barakat, M. Markman and M. E. Randall, Eds., Principles and Practice of Gynecologic Oncology, 5th Edition, Lippincott Williams \& Wilkins, Baltimore, 2009, pp. 683-732.

[3] J. S. Kwon, M. S. Carey, E. F. Cook, F. Qiu and L. F. Paszat, "Addressing Wait Times for Endometrial Cancer Surgery in Ontario," Journal of obstetrics and gynaecology Canada, Vol. 29, No. 12, 2007, pp. 982-987.

[4] K. R. Lee, F. A. Tavassoli, J. Prat, M. Dietel, D. J. Gersell, A. I. Karseladze, et al., "Tumours of the Ovary and Peritoneum," In: F. A. Tavassoli and P. Devilee, Eds., World Health Organization Classification of Tumours. Pathology and Genetics of Tumours of the Breast and Female Genital Organs, IARC Press, Lyon, 2003, pp. 113-145.

[5] P. W. Dickman, A. Sloggett, M. Hills and T. Hakulinen, "Regression Models for Relative Survival," Statistics in Medicine, Vol. 23, No. 1, 2004, pp. 51-64.

doi:10.1002/sim.1597

[6] P. W. Dickman and H. O. Adami, "Interpreting Trends in Cancer Patient Survival," Journal of Internal Medicine, Vol. 260, No. 2, 2006, pp. 103-117. doi:10.1111/j.1365-2796.2006.01677.x

[7] F. Ederer, L. M. Axtell and S. J. Cutler, "The Relative Survival Tart: A Statistical Methodology," National Cancer Institute Monograph, Vol. 6, 1961, pp.101-2.

[8] A. Pokhrel and T. Hakulinen, "How to Interpret the Relative Survival Ratios of Cancer Patients," European Journal of Cancer, Vol. 44, No. 1, 2008, pp. 2661-2667. doi:10.1016/j.ejca.2008.08.016

[9] P. C. Lambert and P. Royston, "Further Development of Flexible Parametric Models for Survival Analysis," The Stata Journal, Vol. 9, No. 2, 2009, pp. 265-290.

[10] P. Royston and M. K. Parmar, "Flexible Parametric Proportional-Hazards and Proportional-Odds Models for Censored Survival Data, with Application to Prognostic Modelling and Estimation of Treatment Effects," Statistics in Medicine, Vol. 21, No. 15, 2002, pp. 2175-2197. doi: $10.1002 /$ sim. 1203

[11] Statistics Canada, "Table 102-0504: Deaths and Mortality Rates, by Age Group and Sex, Canada, Provinces and
Territories, Annual (2112 Series)," Statistics Canada, 2010.

[12] S. Durrleman and R. Simon, "Flexible Regression Models with Cubic Splines," Statistics in Medicine, Vol. 8, No. 5, 1989, pp. 551-561. doi:10.1002/sim.4780080504

[13] Statistics Canada, "Table 051-0001: Population by Sex and Age Group, by Province and Territory," Statistics Canada, 2010. http://www40.statcan.gc.ca/101/cst01/demo31c-eng.htm

[14] C. M. Beard, L. C. Hartmann, G. L. Keeney, C. S. Crowson, G. D. Malkasian, P. C. O'Brien, et al., "Endometrial Cancer in Olmsted County, MN: Trends in Incidence, Risk Factors and Survival," Annals of Epidemiology, Vol. 10, No. 2, 2000, pp. 97-105. doi:10.1016/S1047-2797(99)00039-3

[15] A. Jensen, H. Sharif and S. K. Kjaer, "Use of Fertility Drugs and Risk of Uterine Cancer: Results from a Large Danish Population-Based Cohort Study," American Journal of Epidemiology, Vol. 170, No. 11, 2009, pp. 1408-1414. doi:10.1093/aje/kwp290

[16] A. Klink, L. Tryggvadottir, F. Bray, M. Gislum, T. Hakulinen, H. H. Storm, et al., "Trends in the Survival of Patients Diagnosed with Cancer in Female Genital Organs in the Nordic Countries 1964-2003 Followed up to the End Of 2006," Acta Oncologica, Vol. 49, No. 5, 2010, pp. 632-643. doi:10.3109/02841861003691945

[17] L. Minelli, F. Stracci, S. Prandini, I. F. Moffa and R. F. La, "Gynaecological Cancers in Umbria (Italy): Trends of Incidence, Mortality and Survival, 1978-1998," European Journal of Obstetrics \& Gynecology, Vol. 115, No.1, 2004, pp. 59-65. doi:10.1016/j.ejogrb.2003.11.026

[18] N. K. Lee, M. K. Cheung, J. Y. Shin, A. Husain, N. N. Teng, J. S. Berek, et al., "Prognostic Factors for Uterine Cancer in Reproductive-Aged Women," Obstetrics \& Gynecology, Vol. 109, No. 3, pp. 655-662. doi:10.1097/01.AOG.0000255980.88205.15

[19] L. S. Cook, L. M. Kmet, A. M. Magliocco and N. S. Weiss, "Endometrial Cancer Survival among US Black and White Women by Birth Cohort," Epidemiology, Vol. 17, No. 4, 2006, pp. 469-472. doi:10.1097/01.ede.0000221026.49643.cf

[20] M. E. Sherman and S. S. Devesa, "Analysis of Racial Differences in Incidence, Survival, and Mortality for Malignant Tumors of the Uterine Corpus," Cancer, Vol. 98, No. 1, 2003, pp. 176-186. doi:10.1002/cncr.11484

[21] K. Aoki, J. Sun, A. Kono and J. Misumi, "Age-Related Characteristics of Uterine Cancer Mortality in Japan," Archives of Gynecology and Obstetrics, Vol. 273, No. 2, 2005, pp. 110-114. doi:10.1007/s00404-005-0044-8

[22] L. K. Smith, P. C. Lambert and D. R. Jones, "Up-to-Date Estimates of Long-Term Cancer Survival in England and Wales," British Journal of Cancer, Vol. 89, No. 1, 2003, pp. 74-76. doi:10.1038/sj.bjc.6600976

[23] M. A. Crosby, J. D. Tward, A. Szabo, C. M. Lee and D. K. Gaffney, "Does Brachytherapy Improve Survival in Addition to External Beam Radiation Therapy in Patients with High Risk Stage I and II Endometrial Carcinoma?" American Journal of Clinical Oncology, Vol. 33, No. 4, 2010, pp. 364-349. doi:10.1097/COC.0b013e3181b0c266 
[24] J. M. Straughn, Jr., T. M. Numnum, L. C. Kilgore, E. E. Partridge, J. L. Phillips, M. Markman, et al., "The Use of Adjuvant Radiation Therapy in Patients with Intermediate-Risk Stages IC And II Uterine Corpus Cancer: A Patient Care Evaluation Study from the American College of Surgeons National Cancer Data Base," Gynecologic Oncology, Vol. 99, No. 3, 2005, pp. 530-535. doi:10.1016/i.ygyno.2005.08.034
[25] C. M. Lee, A. Szabo, D. C. Shrieve, O. K. Macdonald and D. K. Gaffney, "Frequency and Effect of Adjuvant Radiation Therapy among Women with Stage I Endometrial Adenocarcinoma," Journal of the American Medical Association, Vol. 295, No 4, 2006, pp. 389-397. doi:10.1001/jama.295.4.389 